\title{
Correlations between Particle Collection Behaviors and Electrohydrodynamics Flow Characteristics in Electrostatic Precipitators
}

\author{
Zhengda Yang ${ }^{1,2 *}$, Hanqing $\mathrm{Li}^{2}$, Qingyi $\mathrm{Li}^{3}$, Riyi Lin ${ }^{1 *}$, Ye Jiang1, Yang Yang ${ }^{2}$, \\ Chenghang Zheng', Deshan $\mathrm{Sun}^{4}$, Xiang Gao ${ }^{2}$ \\ ${ }^{1}$ College of New Energy, Qingdao Engineering Research Center of Efficient and Clean Utilization of Fossil Energy, China \\ University of Petroleum (East China), Qingdao 266580, China \\ ${ }^{2}$ State Key Lab of Clean Energy Utilization, Zhejiang University, Hangzhou 310027, China \\ ${ }^{3}$ Zhejiang Energy Group Co., Ltd., Hangzhou 310007, China \\ ${ }^{4}$ Shandong Guoshun Group, Jinan 250000, China
}

\begin{abstract}
Electrostatic precipitators (ESPs) are widely used to eliminate particulate matter emissions from industrial sources. However, the complex electrohydrodynamics (EHD) flow in ESPs can cause controversial conclusions on particle collection. This paper established an ESP model with an additional source term added to the equation of gas momentum conservation. Correlations between particle motion behaviors and EHD flow characteristics were investigated regarding various discharge voltage, particle size, and electrode position. Results show that the EHD flow presented a back-and-forth distribution between the electrode and plate. The peak value of $v_{y}$ can be as high as $0.15 \mathrm{~m} \mathrm{~s}^{-1}$. The EHD flow can cause four possible types of particle motion status. The dominant factor for particle motion switched between the electric force and drag force along with the particle motion trajectory. There existed an escaping window at the ESP inlet. Particles released from this window penetrated through the ESP rather than be collected. Modifying the ESP geometry and increasing voltage can narrow the escaping window. When the ESP channel width was narrowed from 0.06 to $0.05 \mathrm{~m}$, the maximum $N_{E H D}$ could increase by $73.4 \%$ from 0.94 to 1.63 . Consequently, the collection efficiency was significantly improved. The collection efficiency can even amount to $100 \%$ for particles sized $2.5 \mu \mathrm{m}$.
\end{abstract}

Keywords: Particulate matter; Electrohydrodynamics flow; Electrostatic precipitator; Electric force; Drag force; Particle collection.

\section{INTRODUCTION}

The elimination of air pollutants from power, steel, chemical, and other industries has been a universal requirement to improve the air quality (Chen et al., 2014; Chu et al., 2020; Lin et al., 2020). Among all pollutants, the emission of particulate matter (PM) is one of the thorniest issues. Electrostatic precipitators (ESPs) are the most popular particulate control devices in practical applications due to their high efficiency and low-pressure drop (Yang et al., 2019b). Although the primary principles are understood, fine particles' collection still faces challenges when going through complicated processes, including electrostatics, fluid dynamics, particle charging, and dynamics (Lin et al., 2012; Yang et al., 2020; Yang et al., 2021).

\footnotetext{
* Corresponding authors.

E-mail address: yzd019@zju.edu.cn (Z. Yang);

linry@upc.edu.cn (R. Lin)
}

The electrohydrodynamics (EHD, also called electric wind or ionic wind) is one of the most crucial phenomena in ESPs generated from the momentum transfer between charged ions or particles and the neutral gas molecule (Farnoosh et al., 2011). It reflects the interaction between the primary flow and the secondary flow. The particle drag force can change when a velocity difference exists between the particle and fluid, which subsequently affects the particle migration and collection process (Wang et al., 2019). Some researchers investigated the EHD flow patterns through the particle image velocimetry (PIV) method. Podlinski et al. detected the EHD distribution around the electrode in a pinplate ESP. The EHD was strong at upwind around the tip end and increased with the voltage (Podlinski et al., 2006; Podlinski et al., 2009). Krupa et al. implemented the PIV to study the velocity field of the EHD flow during the back corona discharge and revealed that the EHD flow started from the crater in the ash layer (Krupa et al., 2019).

Additionally, some researchers investigated the EHD flow with the assistance of numerical simulation methods. It was proved that Coulomb repulsion's ion migration was the 
primary reason for EHD flow with the finite difference method (Kawamoto et al., 2006). The generation of EHD flow in the ESP can also be predicted through the finite volume method (Neimarlija et al., 2011). EHD characteristics can be affected by many factors. Li et al. studied the effects of temperature on the EHD flow with a computational fluid dynamics (CFD) model. The results showed that high gas temperature caused more significant ionic wind effects on the secondary gas flow (Li et al., 2015). Particle concentration can influence the gas flow pattern and corona discharge current regardless of mono-dispersed or poly-dispersed size distribution (Farnoosh et al., 2010; Farnoosh et al., 2011).

Up to now, the impacts of complex EHD on particle collection are still controversial. Some researchers believed that dust re-entrainment could occur when the EHD acted on the dust layers on the collection plate, which deteriorated the particle collection performance (Soldati, 2000). In contrast, other researchers believed that EHD flow could be taken advantage of to improve collection efficiency. Yamamoto $e t$ al. added a dust collector on the dust collector plate so that particles can be blown into the dust collector through with ionic wind, which helped to reduce the dust re-entrainment and enhanced particle collection efficiency (Yamamoto et al., 2009; Yamamoto et al., 2010). The ESP performance could also be improved by using an ionic-wind-assisted pre-charger because particle charging and coagulation were enhanced by electric force or turbulence (Chang et al., 2015).

Although many achievements have been made upon the EHD flow in ESPs, its influence on particle collection is still not apparent. The above-mentioned work's primary debate was ascribed to the dust re-entrainment, which was an abnormal phenomenon in dry ESPs. In wet ESPs, this issue can be simplified because dust re-entrainment no longer existed because of the water film on plates. In addition, the effects of EHD flow were mainly deduced from the change of total efficiency or fractional efficiency, no matter by experiments or simulations. These efficiencies were derived from statistically averaged concentrations, which cannot reflect the particle movement through the whole ESP channel. Especially, the distribution of ionic wind in ESPs was not uniform in space. It depended on electrode configuration, operation voltage, and particle size. Its influence on particle collection was likely to change in different regions.

This paper established a three-electrode ESP to investigate the correlations between particle collection behaviors and EHD flow characteristics. Three typical particle sizes, i.e., $0.1 \mu \mathrm{m}, 1 \mu \mathrm{m}$, and $2.5 \mu \mathrm{m}$, were selected because they are the critical size for $\mathrm{PM}_{0.1}, \mathrm{PM}_{1.0}$, and $\mathrm{PM}_{2.5}$, respectively. The distribution of EHD flow, particle forces, particle dispersion, and particle deposition were investigated systematically from the whole channel or particle migration process. Finally, two electrode configurations were compared to find out possible retrofitting approaches.

\section{NUMERICAL METHODS}

\section{Corona Discharge}

During the ESP operation process, the discharge electrode is connected with a high voltage power source. The electrostatic field is established, and considerable ions are generated within the ESP channel space. The Poisson equation in Eq. (1) (Farnoosh et al., 2010) was used to describe the electrostatic field, the distribution of electric potential, and charge density. Accordingly, the electric field strength can be obtained by calculating the gradient's gradient with Eq. (2). The electric field strength on the surface of the discharge electrode can be determined concerning the Peek's formula given in Eq. (3) (Luo et al., 2015) because it is regarded constant after the initial onset of corona discharge.

$\nabla^{2} \varphi=-\frac{\rho_{\text {ion }}+\rho_{p}}{\varepsilon_{0}}$

$E=-\nabla \varphi$

$E_{S}=E_{0} m\left(\delta+0.0308 \sqrt{\frac{\delta}{r_{0}}}\right)$

The corona current is generated when ions transport from the discharge electrode towards the collection plates under the Coulomb force's effects. The corona current density is comprised of three components, i.e., drift current, convection current, and diffusion current, as shown in Eq. (4). Typically, the drift current dominates the overall corona current. Thus, the equation can be further simplified to Eq. (5). During the ion transportation, current continuity is exactly satisfied according to Eq. (6) (Farnoosh et al., 2010).

$J=\rho_{\text {ion }} k_{\text {ion }} E+\rho_{\text {ion }} u-D_{i} \nabla \rho_{\text {ion }}$

$J=\rho_{i o n} k_{i o n} E$

$\nabla J=\nabla\left(\rho_{\text {ion }} k_{\text {ion }} E\right)=0$

\section{Particle Charging Model}

The prerequisite for electrostatic separation is that particles should be electrically charged. Many researchers have developed the particle charging theory. The field modified diffusion (FMD) model proposed by Lawless (1996) was widely employed to calculate the particle charging. This theory accounts for the field charging and additional diffusion charging. It is expressed with a linear differential equation, which must be solved with respect to time. The original form given by Lawless was dimensionless. Schmidconverted it into a more understandable form (Hans-Joachim and Hans, 1998; Schmid and Vogel, 2003), as shown in Eq. (7), when modeling particle transport in electrostatic precipitators.

$$
\frac{d q_{p}}{d t}= \begin{cases}\frac{q_{s}}{\tau}\left(1-\frac{q}{q_{s}}\right)^{2}+\frac{2 \pi \alpha \rho_{i o n} k_{i o n} k_{B} d_{p}}{e} & q \leq q_{s} \\ \frac{\alpha}{4 \tau} \frac{\left(q-q_{s}\right)}{\exp \left(\frac{e\left(q-q_{s}\right)}{2 \pi \varepsilon_{0} k_{i o n} k_{B} T d_{p}}\right)-1} & q>q_{s}\end{cases}
$$


The overall charging rate given in Eq. (7) simultaneously accounts for field charging and additional diffusion charging. As for field charging, there exists a saturation charge amount at a given electric field strength. Particles acquire charges by field charging only when the actual particle charge is smaller than the saturation charge. In contrast, diffusion charging still makes sense when the actual particle charge is above the saturation value (Mizuno, 2000). The $q_{s}$ in Eq. (7) represents the saturation particle charge for field charging, which can be calculated with Eq. (8).

$q_{s}=3+\frac{\varepsilon_{r}}{\varepsilon_{r}+2} \pi \varepsilon_{0} d_{p}^{2} E$

The $\alpha$ and $e_{\text {norm }}$ in Eq. (7) are model constants, which can be calculated with Eq. (9) and Eq. (10), respectively.

$\alpha= \begin{cases}1 & e_{\text {norm }}<0.525 \\ \frac{1}{\left(e_{\text {norm }}+0.457\right)^{0.575}} & e_{\text {norm }}>0.525\end{cases}$

$e_{\text {norm }}=\frac{e d_{p}}{2 k_{B} T} E$

\section{Gas Flow Dynamics}

In industrial conditions, the flue gas can be reasonably treated as an incompressible fluid. In this paper, the gas flow was simulated under turbulent and steady conditions. Compared with neutral gases, the gas flow dynamics is of considerable difference when corona discharge is exerted. The ionized gas drifted towards the collection plates driven by the electric force, and the EHD flow is generated consequently. Governing equations of mass conservation and momentum conservation shown in Eqs. (11) and (12) are used to describe gas flow dynamics. The additional source term $\rho_{\text {ion }} E$ in Eq. (12) represents such electric force effects on the flow field.

$$
\nabla\left(\rho_{\text {gas }} u\right)=1
$$

$\rho_{g a s}\left(\frac{\partial u}{\partial t}+u \cdot \nabla u\right)=-\nabla P+\mu_{e f f} \nabla^{2} u+\rho_{\text {ion }} E$

\section{Particle Motion Dynamics}

Generally, particle motion can be driven by several forces, including drag force, Coulomb force, gravitational force, buoyancy force, Brownian force, Saffman lift force, thermophoretic force, pressure gradient force, and virtual mass force. Under ESP conditions, however, the particle behaviors prove to be mainly dominated by drag force and Coulomb force (Li et al., 2015). Besides, the particle flow in an ESP is generally treated as dilute flow (Gao et al., 2020). Interactions among particles and between wall and particles can be reasonably neglected (Kallio and Stock, 1992). Accordingly, the governing equation of particle motion in an ESP can be simplified as: $m_{p} \frac{d u_{p}}{d t}=\frac{1}{8} \pi C_{d} \rho_{g a s} d_{p}^{2}\left|u-u_{p}\right|\left(u-u_{p}\right)+q E$

where the drag force coefficient can be defined as (Lu et al., 2016):

$C_{d}= \begin{cases}\frac{18}{\mathrm{Re}} & \mathrm{Re}<1 \\ \frac{22.73}{\mathrm{Re}}+\frac{0.0903}{\mathrm{Re}^{2}}+3.69 & 0.1<\mathrm{Re}<1\end{cases}$

When the particle size is comparable with the molecular mean free path, the drag force should be corrected by dividing the Cunningham correction factor defined as follows (Ounis et al., 1991):

$C m=1+\frac{2 \lambda}{d_{p}}\left(1.257+0.4 \exp \left(-\frac{1.1 d_{p}}{2 \lambda}\right)\right)$

\section{MODELING PROCEDURE}

The commercial FLUENT CFD software package assisted with user-defined functions (UDFs) is used for the modeling solution. In this paper, the RNG k-epsilon model and standard wall functions were selected to solve gas flow equations. The Lagrangian approach was implemented to describe the particle charging and particle motion through an ESP. The electric field, ion density, particle charging, and ionic wind were embedded with separate UDFs. Besides the convergence of all governing equations, the convergence of electric field strength on the electrode surface should also be guaranteed. The accuracy of this model has been verified several times in previous work (Yang et al., 2018a). In this paper, a horizontal ESP was used to investigate particle motion behavior and EHD flow characteristics. The particle concentration and gas velocity were $50 \mathrm{mg} \mathrm{m}^{-3}$ and $1 \mathrm{~m} \mathrm{~s}^{-1}$, respectively, similar to the experimental conditions (Yang et al., 2018b). The geometry was simplified to a typical threeelectrode arrangement, as shown in Fig. 1. The electrode space $(s)$ and the channel width $(w)$ were $0.1 \mathrm{~m}$ and $0.06 \mathrm{~m}$, respectively. The computational domain was symmetrically treated to save computational resources because the modeling was done under steady conditions. As shown in Fig. 1, the geometry was discretized with unstructured mesh, and the regions around the wall boundary were refined to ensure modeling accuracy. Compared with dry ESPs, it is ideal to set the collection plate of wet ESPs as a trap boundary condition because the water film can ensure no reflection occurs after particle capture.

\section{RESULTS AND DISCUSSION}

\section{EHD Flow Characteristics}

The ionic wind acts as an external disturbance to the primary gas flow, affecting the ESP channel's overall gas flow pattern. In this study, the ionic wind can be characterized by the component of gas velocity y-direction $\left(v_{y}\right)$. Fig. 2 


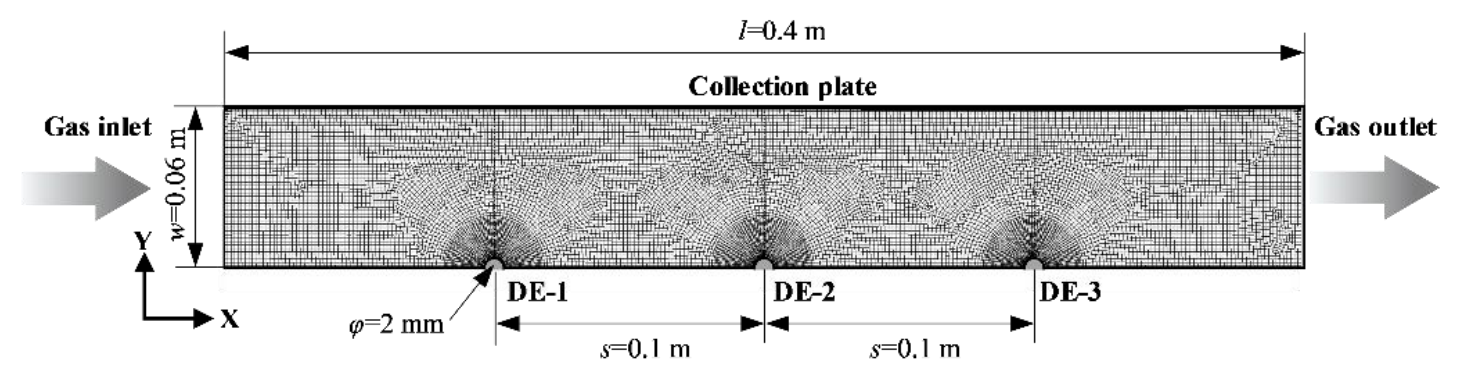

Fig. 1. The geometry of the three-electrode ESP and geometry meshing.
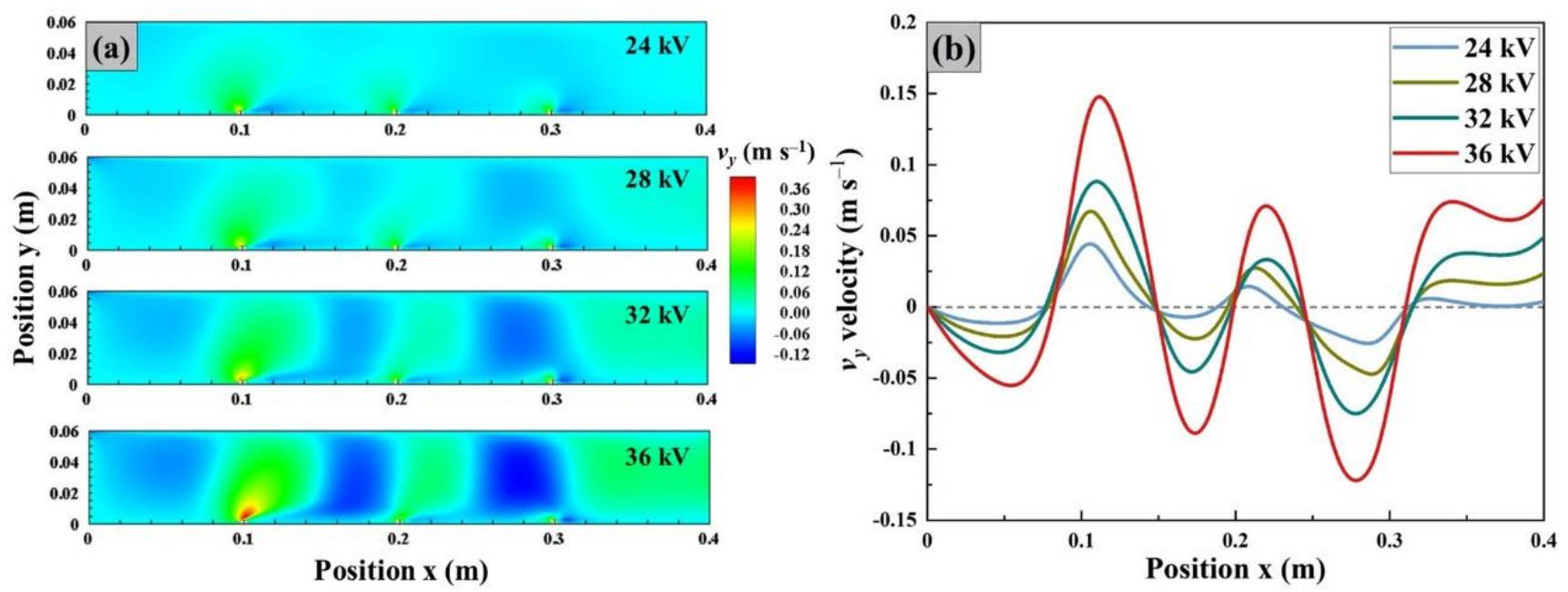

Fig. 2. Distribution of $v_{y}$ under discharge voltages ranging from 24 to $36 \mathrm{kV}$, (a) $2 \mathrm{D}$ contour, (b) data at the line of $y=0.03 \mathrm{~m}$.

shows the distribution of $v_{y}$ under different discharge voltages. It can be seen that the disturbance of ionic wind to the primary flow gas became more significant as the discharge voltage increased from 24 to $36 \mathrm{kV}$. The ionic wind was generated from the discharge electrode's surface, where the space charge was released. Subsequently, the gas was driven by the ionic wind from the electrode towards the collection plate. When the flowing gas reached the collection plate, it reversed back towards the electrode. Fig. 2(b) shows, as an example, the back-and-forth flow along with the line of $y=$ $0.03 \mathrm{~m}$ under different discharge voltages. The peak value of $v_{y}$ was as high as $0.15 \mathrm{~m} \mathrm{~s}^{-1}$, almost equivalent to the effective particle migration velocity (Yang et al., 2017). This ionic wind may lead to a high risk of dust re-entrainment in dry ESPs (Soldati, 2000), but the water film in wet ESPs can overcome this disadvantage. Instead, the high-velocity ionic wind may reduce the resistance to particle motion. However, the reverse flow's negative effect cannot be neglected because it may increase the resistance to particle motion. A more detailed analysis can be obtained by particle motion and forces.

\section{Particle Force Analysis}

Inside an ESP, the particle motion depends on comprehensive external electric and flow field conditions. Fig. 3 shows the components of particle velocity and forces in y-direction along with the particle motion trajectory. For simplicity, the subsequent statements of particle velocity, drag force, and electric force refer to the component rather than the overall ones. Three particle sizes $\left(d_{p}=0.1 \mu \mathrm{m}\right.$, $1.0 \mu \mathrm{m}, 2.5 \mu \mathrm{m}$ ) were analyzed as typical cases. The particle released from $y=0.003 \mathrm{~m}$ was tracked as an example. As can be seen, the particle forces presented a similar tendency for particles of different sizes. The drag force and electric force varied simultaneously along with the motion trajectory, and they always tried to maintain a balance. They reached a peak around the electrode's position $(x=0.1 \mathrm{~m}, 0.2 \mathrm{~m}, 0.3 \mathrm{~m})$, and approached a bottom at the space between two electrodes. Particles always tend to be accelerated or decelerated to reach the balance of external forces. According to the matching of particle velocity and forces in Fig. 3(c), the particle status can be classified into four possible types: (A) Particles were decelerated with the decreasing electric force; (B) Particles were decelerated with the increasing drag force; (C) Particles were accelerated with the increasing electric force; (D) Particles were accelerated with the decreasing drag force. The drag force always acted as resistance to particle motion. The ionic wind can exert an effect on particle motion through the changing of motion resistance. The variations in electric force and drag force can be attributed to the positiondependent electric field and flow field. Therefore, the dominant factor for particle motion switched along with the particle motion trajectory.

\section{Multi-particle Motion}

The particle motion presented considerable complexity 

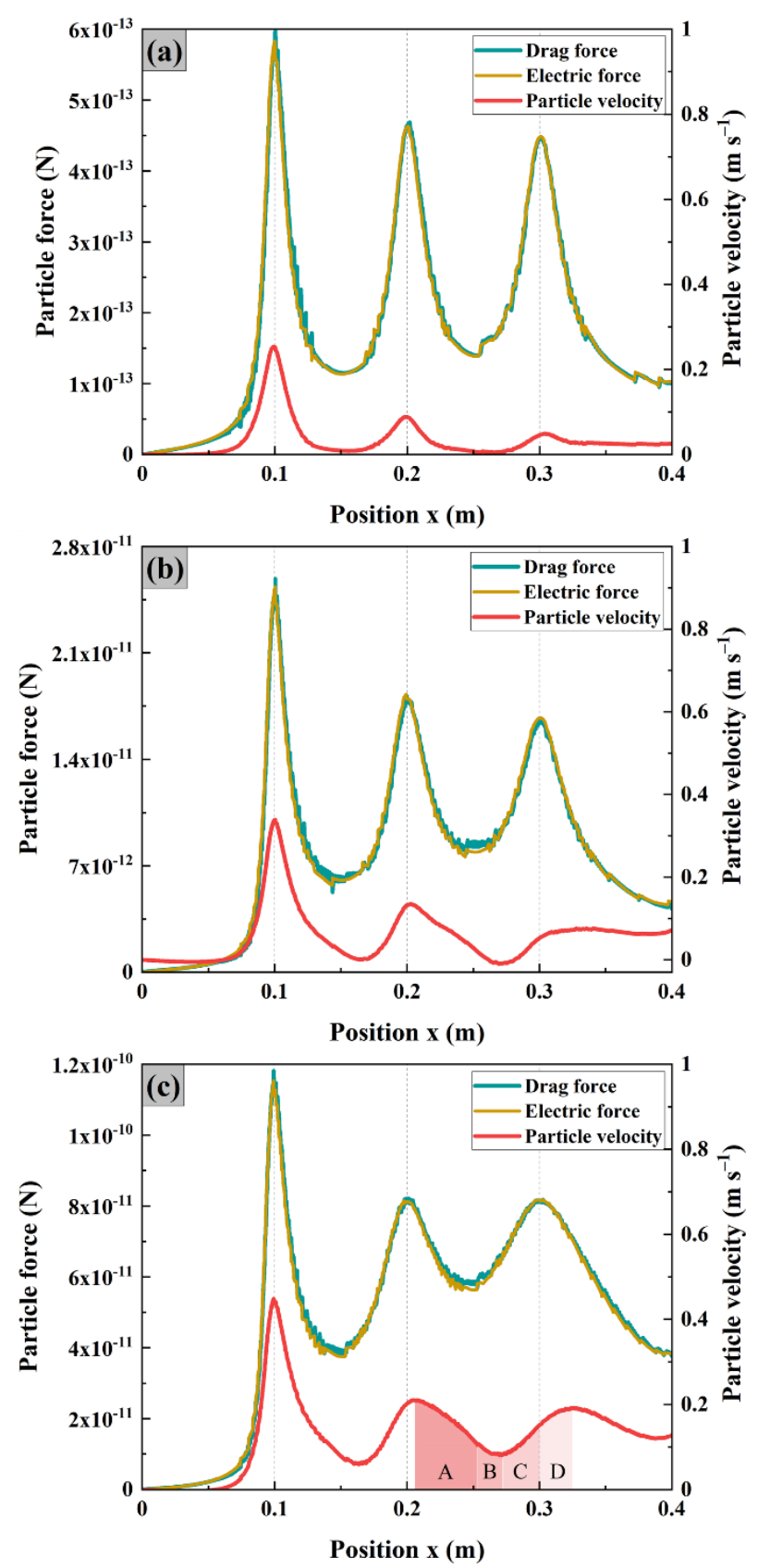

Fig. 3. The drag force and electric force of different particle sizes (a) $0.1 \mu \mathrm{m}$, (b) $1.0 \mu \mathrm{m}$, and (c) $2.5 \mu \mathrm{m}$ along with the particle motion trajectory. (Discharge voltage, $U=32 \mathrm{kV}$; particle release position, $y=0.003 \mathrm{~m}$ )

under the coupled flow field and electric field. Particles can have different trajectories and charging rates due to the nonuniform electric field and space charge density. Notably, the dispersion and deposition patterns of multi-particle can be influenced by the external fields in the whole channel space.

\section{Particle Dispersion Pattern}

The overall and grade collection efficiencies only reflect the averaged results of all injection particles. Whereas, the particle dispersion pattern in the space can help analyze which particles are the most likely to escape from the ESP.
Fig. 4 shows the dispersion pattern of three particle sizes, and they were pictured under the same residence time. When the gas approached the first electrode $(t=0.1 \mathrm{~s})$, particles with different sizes presented a similar linear pattern. Most particles were dragged around the line corresponding to the first electrode for the three cases. Only a few particles were
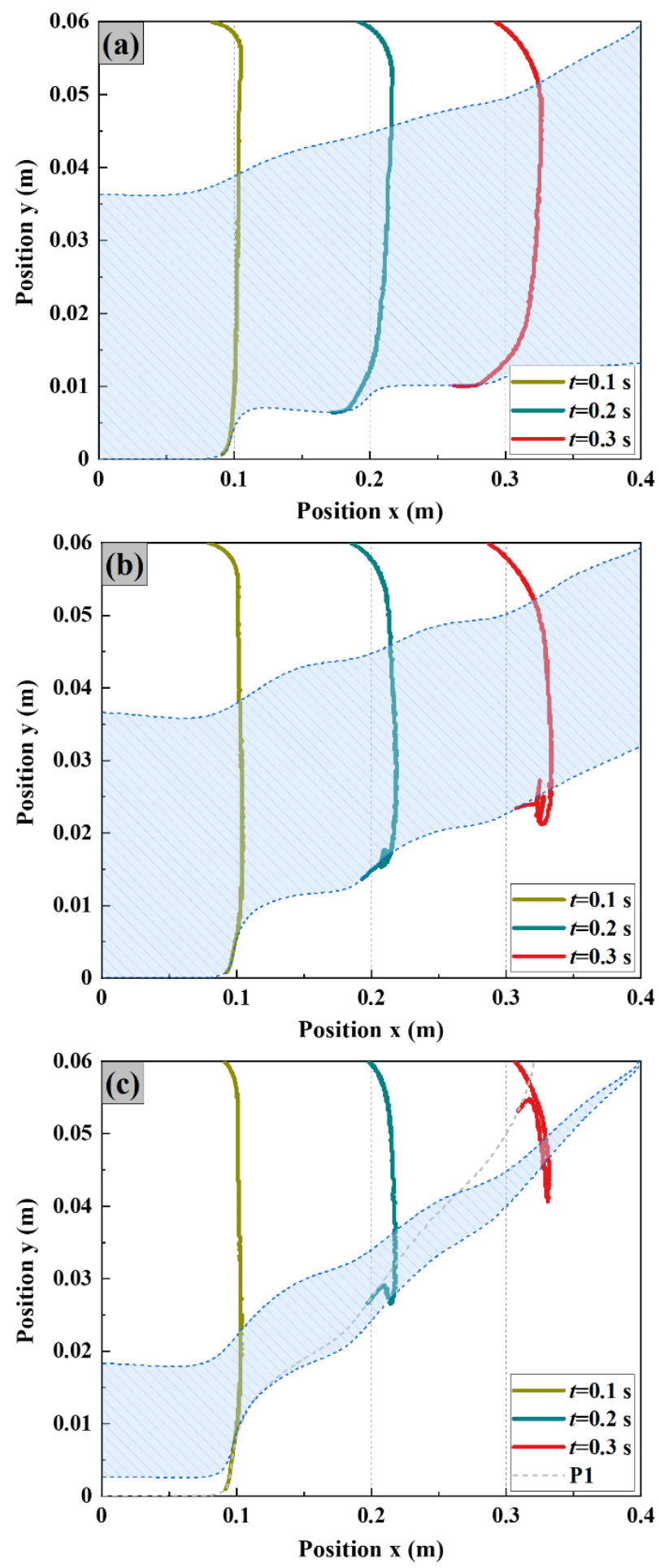

Fig. 4. Particle dispersion pattern of different particle sizes (a) $0.1 \mu \mathrm{m}$, (b) $1 \mu \mathrm{m}$, (c) $2.5 \mu \mathrm{m}$. (Discharge voltage, $U=$ $32 \mathrm{kV}$ ) 
left behind this line. The boundary layer along the plate hindered the particles near the collection plate. The electric force in $\mathrm{x}$-direction changed from positive to negative when the particle passed the electrode, which also can hinder the particle motion. When the gas approached the second electrode $(t=0.2 \mathrm{~s})$, particles' dispersion patterns with different sizes differed obviously. For particle-sized $1.0 \mu \mathrm{m}$ and $2.5 \mu \mathrm{m}$, a part of particles near the electrode broke away from the original sequence. With the increasing particle size, particle charging tended to be primarily dominated by field charging. When particles passed through the area with high electric field strength and ion density, they were driven by the high electric force. This difference became significant when the gas approached the third electrode $(t=0.3 \mathrm{~s})$. Particles near the electrode (the farthest from the plate) were even easier to be captured than those away from the electrode for particles sized $2.5 \mu \mathrm{m}$. For example, the particle released from $y=$ $0.00012 \mathrm{~m}$ (labeled P1) was captured at $x=0.32 \mathrm{~m}$.

The net result of the above-mentioned process produced an escaping window, which corresponded to the shaded region in Fig. 4. Particles released from this window penetrated through the ESP rather than be collected. Particle escaping characteristics relied on three conditions, i.e., particle injection position, particle size, and external fields. Given stable external fields, particles near the electrode went through longer migration distance than that near the plate, but meanwhile, the charging condition was better for them. Consequently, the escaping window tended to be narrowed from both sides when the particle size increased from $0.1 \mu \mathrm{m}$ to $2.5 \mu \mathrm{m}$. When the tracked particle size was $2.5 \mu \mathrm{m}$, only particles injected from $y=0.00265 \mathrm{~m}$ to $y=0.0183 \mathrm{~m}$ escaped from the ESP. The corresponding collection efficiency was as high as $74 \%$. The escaping window variation was insignificant for particles sized $0.1 \mu \mathrm{m}$ and $1.0 \mu \mathrm{m}$ because their drag force was corrected by Cunningham correction (Yang et al., 2018a). Similarly, particles in $0.1-1.0 \mu \mathrm{m}$ present a U-shape curve of removal efficiency. However, the escaping window for particles sized $1.0 \mu \mathrm{m}$ can be further narrowed if the effective length of the ESP channel can be extended.

The particle motion can be further analyzed in two separate directions to get an insight into the particle dispersion pattern. The particle residence time (before collection or escape) and the averaged particle migration velocity were used to characterize the fate of particles released from different positions (Fig. 5). The marked ranges I, II, and III in Fig. 5(a) exactly corresponded to the escaping window in Fig. 4. The particle released from the critical trap position would be exactly collected at $x=0.4 \mathrm{~m}$ and $y=0.06 \mathrm{~m}$. The residence time for particles released from the escaping window increased as the particle release position approached to the critical trap position. For particles outside this window but near the plate, the particle residence time decreased as the particle release position was close to the collection plate. Although the particles near the plate were easy to capture, they presented low migration velocity according to Fig. 5(b), which can be ascribed to the insufficient particle charging. For particles outside these ranges but near the electrode (only applicable to particles sized $2.5 \mu \mathrm{m}$ ), the residence time decreased with the increasing migration distance instead. They presented high migration velocity according to Fig. 5(b), which can be attributed to the sufficient particle charging. In general, the particle collection process both depended on migration distance and migration velocity. Different strategies can be taken to improve the removal efficiency of particles with different particle sizes. For fine particles, modifying the ESP geometry is expected to improve particle collection efficiency. Due to particle charging's inherent limitations, it is not suggested to improve the collection efficiency only by enhancing particle charging. As for coarse particles, the enhancement of particle charging is effective to improve removal efficiency.

\section{Particle Deposition Pattern}

The particle deposition pattern reflects the results after
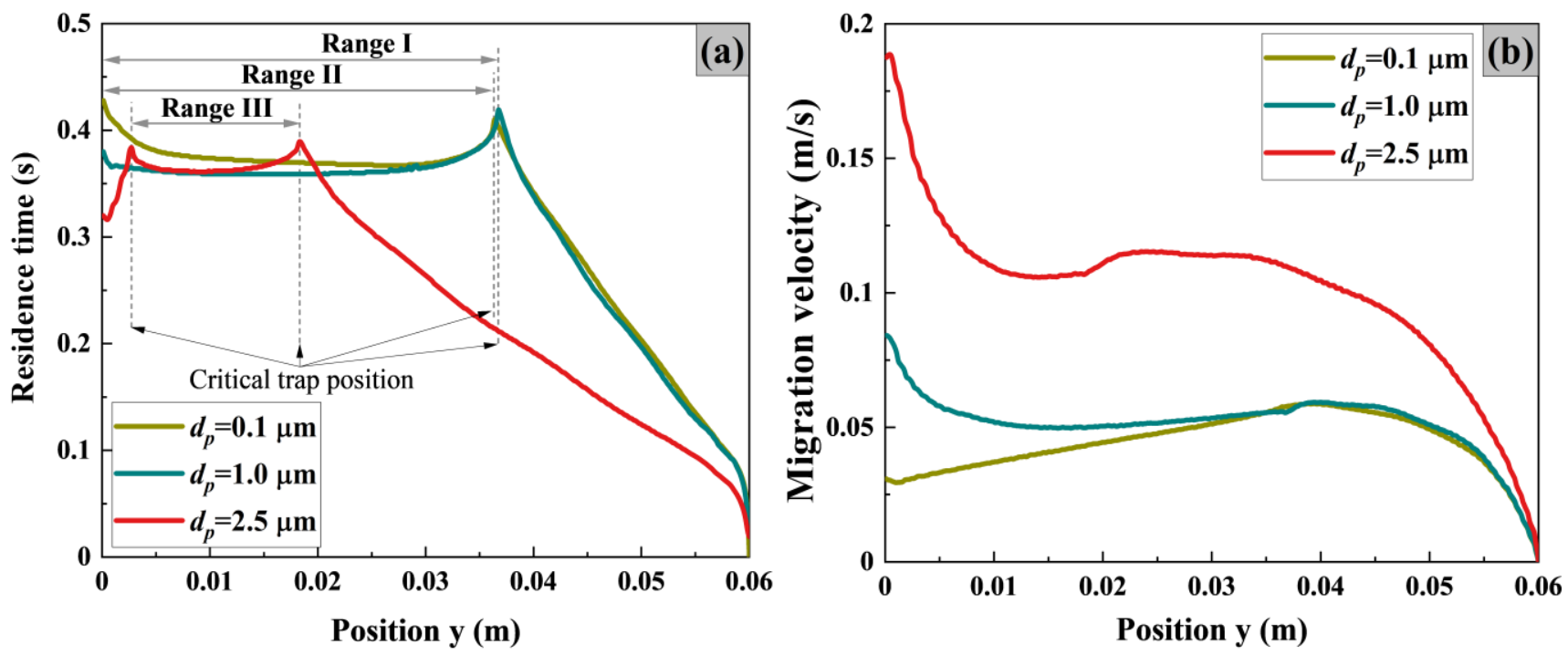

Fig. 5. (a) Residence time before collection or escaping, (b) averaged migration velocity of particle released from different positions. 


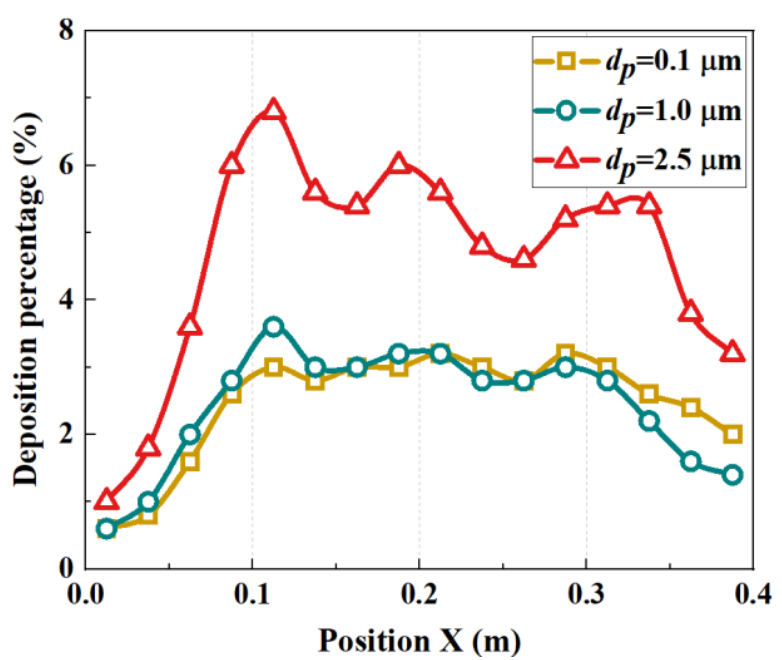

Fig. 6. Particle deposition pattern of particle-sized $0.1 \mu \mathrm{m}$, $1.0 \mu \mathrm{m}$, and $2.5 \mu \mathrm{m}$.

collection, the understanding of which is beneficial for plate cleaning and electrode arrangement. Fig. 6 shows the percentage of particles with three different sizes on the collection plates in 16 intervals along the length in the $\mathrm{x}$ direction. The deposition percentage represents the deposition probability of a specific position in the x-direction. As can be seen, particles began to deposit as soon as they entered the ESP. The deposition probability increased considerably until the particle reached the first electrode. As expected, the deposition probability was high in areas corresponding to each electrode due to the strong electric forces. Regardless of particle size, the deposition probability was reduced in areas between the two electrodes because the electric field strength was weak in these areas. With the flow of flue gas, the particle concentration decreased along the ESP channel. Therefore, the deposition probability for particles sized
$1.0 \mu \mathrm{m}$ and $2.5 \mu \mathrm{m}$ decreased gradually along the plate length. For example, the peak deposition probability for particles of $2.5 \mu \mathrm{m}$ decreased from $6.8 \%$ to $5.4 \%$ at the position corresponding to the first and third electrodes. The deposition probability agrees with the dust accumulation on the deposition planes in actual application. However, the deposition probability for particles of $0.1 \mu \mathrm{m}$ was less affected by the electrode's position, and its deposition probability was relatively uniform along the plate length. The Stokes number of particle-sized $0.1 \mu \mathrm{m}$ was far less than 1 . Thus, its motion was more likely affected by the drag from the gas flow.

\section{Particle Collection Efficiency}

Although particle dispersion and deposition patterns are the direct evidence of particle collection, the collection efficiency gains more popularity in practical applications. The efficiency data are presented in Fig. 7 as a function of a dimensionless $N_{E H D}$ number, which can be calculated with $N_{E H D}=\left(j_{C E} 2 w\right) /\left(b_{i} \rho_{\text {gas }} u^{2}\right)$. As can be seen from Fig. $7(\mathrm{a})$, the particle collection efficiency increased with the increasing $N_{E H D}$ when the $N_{E H D}$ was a result of increasing voltage. For example, the collection efficiency of 1-micron particle increased from $14.0 \%$ to $51.8 \%$, with the $N_{E H D}$ increasing from 0.10 to 0.92 . The ionic wind showed positive net effects on particle collection. Notably, the effects of $N_{E H D}$ on collection efficiency can be significantly affected by the particle size. The collection efficiency was almost kept constant when the particle size increased from $0.1 \mu \mathrm{m}$ to $1.0 \mu \mathrm{m}$. In contrast, the collection efficiency increased from $38.4 \%$ to $74.2 \%$ when the particle size increased from $1.0 \mu \mathrm{m}$ to $2.5 \mu \mathrm{m}$ under a constant $32 \mathrm{kV}$ voltage. This difference can be ascribed to that charged particles can generate a stronger secondary electric field. Particles with different sizes exert various effects of this corona suppression (Yang et al., 2018a, 2019a).

In addition to particle size and discharge voltage, two
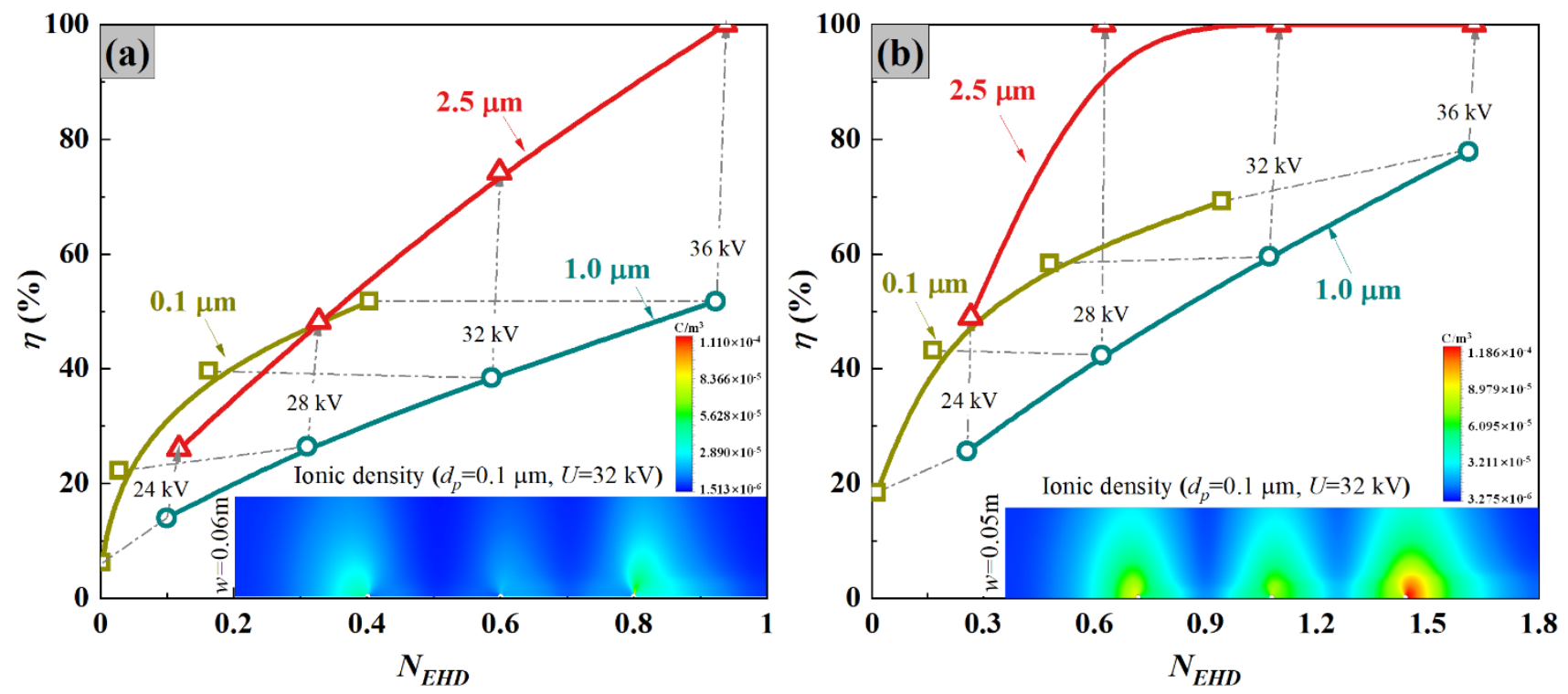

Fig. 7. Particle collection efficiency of two electrode configurations under different $N_{E H D}$ numbers, (a) $w=0.06 \mathrm{~m}$, and (b) $w$ $=0.05 \mathrm{~m}$. 
electrode configurations are compared to find out possible retrofitting approach. The channel width was set as $w=$ $0.06 \mathrm{~m}$, and $w=0.05 \mathrm{~m}$ since the particle motion relies on the particle injection position. As can be seen, when the channel width was reduced to $0.05 \mathrm{~m}$, the collection efficiency presented a similar tendency but with a noticeable improvement. Even all particles with a size of $2.5 \mu \mathrm{m}$ can be collected $(\eta=100 \%)$ when the discharge voltage exceeded $28 \mathrm{kV}$. This result can be attributed to the narrowing channel width is beneficial for inhabiting corona suppression and enhancing corona discharge. Consequently, the maximum $N_{E H D}$ number increased by $73.4 \%$ even under the same discharge voltage and particle size. The contour graphics in Fig. 7 represent the distribution of ionic density, and it can be seen the increase in discharge intensity when the channel width was reduced to $0.05 \mathrm{~m}$. Therefore, the narrowing channel width reduces the migration distance and improves the collection efficiency by inhabiting corona suppression.

\section{CONCLUSIONS}

This work focuses on determining the correlations between particle motion behaviors and the complex EHD flow characteristics in the ESP. The EHD flow presented a back-and-forth distribution between the electrode and the plate. The particle motion status can be classified into four possible types under the effects of EHD flow. The dominant factor for particle motion switched along with the particle motion trajectory. Particles released from the escaping window penetrated through the ESP rather than be collected. The residence time for particles released from the escaping window increased as the particle release position approached the critical trap position. This window was related to migration distance and migration velocity. Modifying the ESP geometry is expected to improve fine particle collection while increasing particle charge amount is expected to enhance coarse particle collection. The deposition probability was high in areas corresponding to each electrode, but the deposition probability for particles of $0.1 \mu \mathrm{m}$ was less affected by the electrode's position. The collection efficiency increased with the increasing $N_{E H D}$ when the $N_{E H D}$ was a result of increasing voltage. When the channel width was narrowed from 0.06 to $0.05 \mathrm{~m}$, the maximum $N_{E H D}$ could increase by $73.4 \%$ from 0.94 to 1.63. Consequently, the collection efficiency was significantly improved, and the efficiency can even amount to $100 \%$ for particles sized $2.5 \mu \mathrm{m}$.

\section{NOMENCLATURE}

$b \quad$ ionic mobility, $\mathrm{cm}^{2}(\mathrm{~V} \mathrm{~s})^{-1}$

$C_{d} \quad$ drag coefficient

$C_{m} \quad$ Cunningham correction factor

$D_{i} \quad$ ion diffusion coefficient, $\mathrm{m}^{2} \mathrm{~s}^{-1}$

$d_{p} \quad$ particle diameter, $\mathrm{m}$

$E \quad$ electric field strength, $\mathrm{V} \mathrm{m}^{-1}$

Es electric field strength on the electrode surface, $\mathrm{V} \mathrm{m}{ }^{-1}$

$E_{0} \quad$ breakdown electric field strength, $\mathrm{V} \mathrm{m}^{-1}$

$e \quad$ electronic charge, $1.6 \times 10^{-19} \mathrm{C}$

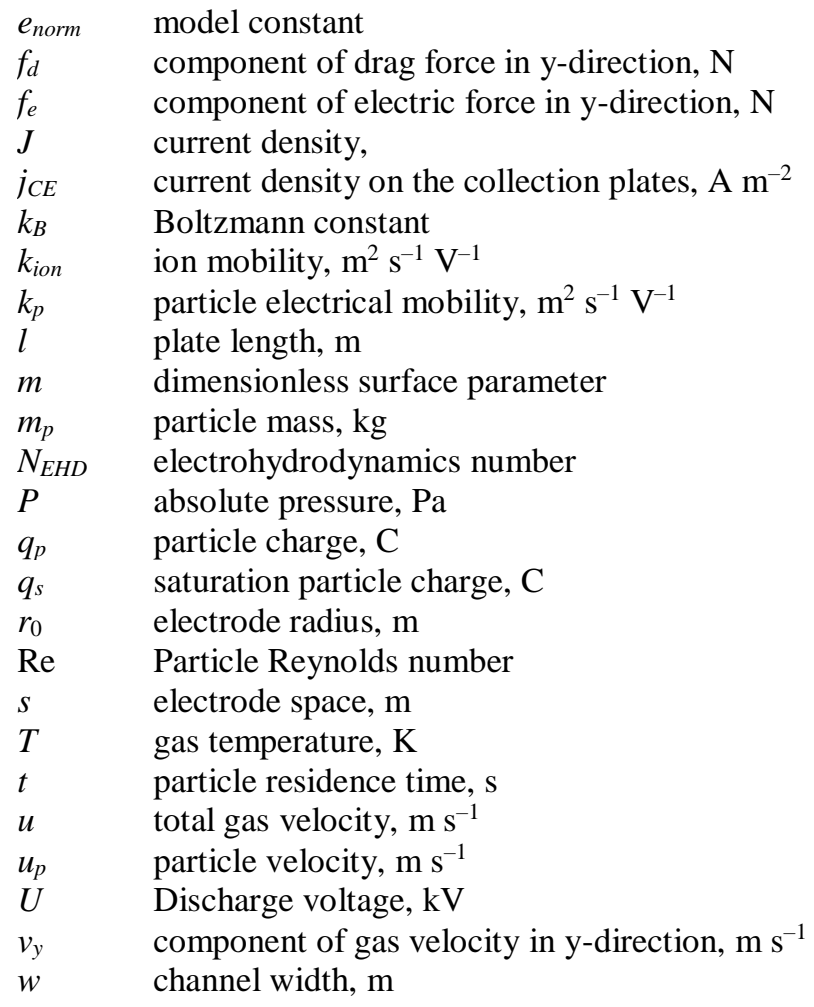

\section{Greek Symbols}

$\alpha \quad$ model constant

$\delta \quad$ relative gas density

$\varepsilon_{0} \quad$ permittivity of free space, $\mathrm{C} \mathrm{V}^{-1} \mathrm{~m}^{-1}$

$\varepsilon_{r} \quad$ relative permittivity

$\eta \quad$ collection efficiency, $\%$

$\lambda$ mean free path, $m$

$\mu_{e f f} \quad$ effective gas viscosity, $\mathrm{kg} \mathrm{m}^{-1} \mathrm{~s}^{-1}$

$\rho_{p} \quad$ space charge density of particle, $\mathrm{C} \mathrm{m}^{-3}$

$\rho_{\text {ion }} \quad$ space charge density of ion, $\mathrm{C} \mathrm{m}^{-3}$

$\rho_{\text {gas }} \quad$ gas density, $\mathrm{kg} \mathrm{m}^{-3}$

$\tau \quad$ time constant of field charging, $\mathrm{s}$

$\varphi \quad$ electric potential, $\mathrm{V}$

\section{ACKNOWLEDGMENTS}

This work was supported by the National Natural Science Foundation of China (No. 51906258), and Key Research and Development Program of Shandong Province (No. 2019JZZY010403).

\section{DISCLAIMER}

The authors declare no conflicts of interest.

\section{REFERENCES}

Chang, Q., Zheng, C., Gao, X., Chiang, P., Fang, M., Luo, Z. and Cen, K. (2015). Systematic approach to optimization of submicron particle agglomeration using ionic-windassisted pre-charger. Aerosol Air Qual. Res. 15: 27092719. https://doi.org/10.4209/aaqr.2015.06.0418

Chen, T.M., Tsai, C.J., Yan, S.Y. and Li, S.N. (2014). An 
efficient wet electrostatic precipitator for removing nanoparticles, submicron and micron-sized particles. Sep. Purif. Technol. 136: 27-35. https://doi.org/10.1016/j.sep pur.2014.08.032

Chu, F., Su, M. and Yang, G. (2020). Heat and mass transfer characteristics of ammonia regeneration in packed column. Appl. Therm. Eng. 176: 115405. https://doi.org/ 10.1016/j.applthermaleng.2020.115405

Farnoosh, N., Adamiak, K. and Castle, G.S.P. (2010). 3-D numerical analysis of EHD turbulent flow and monodisperse charged particle transport and collection in a wire-plate ESP. J. Electrostat. 68: 513-522. https://doi.org/10.1016/j.elstat.2010.07.002

Farnoosh, N., Adamiak, K. and Castle, G.S.P. (2011). 3-d numerical simulation of particle concentration effect on a single-wire ESP performance for collecting polydispersed particles. IEEE Trans. Dielectr. Electr. Insul. 18: 211-220. https://doi.org/10.1109/tdei.2011.5704512

Gao, W.C., Wang, Y.F., Zhang, H., Guo, B.Y., Zheng, C.H., Guo, J., Gao, X. and Yu, A.B. (2020). A numerical investigation of the effect of dust layer on particle migration in an electrostatic precipitator. Aerosol Air Qual. Res. 20: 166-179. https://doi.org/10.4209/aaqr.2019.11.0609

Hans-Joachim, S. and Hans, B. (1998). On the modeling of particle transport in electrostatic precipitators. Proceedings of $7^{\text {th }}$ International Conference on Electrostatic Precipitation. pp. 673-687.

Kallio, G.A. and Stock, D.E. (1992). Interaction of electrostatic and fluid dynamic fields in wire-plate electrostatic precipitators. J. Fluid Mech. 240: 133-166. https://doi.org/10.1017/s0022112092000053

Kawamoto, H., Yasuda, H. and Umezu, S. (2006). Flow distribution and pressure of air due to ionic wind in pinto-plate corona discharge system. J. Electrostat. 64: 400407. https://doi.org/10.1016/j.elstat.2005.10.023

Krupa, A., Podlinski, J., Mizeraczyk, J. and Jaworek, A. (2019). Velocity field of EHD flow during back corona discharge in electrostatic precipitator. Powder Technol. 344: 475-486. https://doi.org/10.1016/j.powtec.2018.12. 006

Lawless, P.A. (1996). Particle charging bounds, symmetry relations, and an analytic charging rate model for the continuum regime. J. Aerosol Sci. 27: 191-215. https://doi.org/10.1016/0021-8502(95)00541-2

Li, Y., Zheng, C., Luo, K., Gao, X., Fan, J. and Cen, K. (2015). CFD simulation of high-temperature effect on EHD characteristics in a wire-plate electrostatic precipitator. Chin. J. Chem. Eng. 23: 633-640. https://doi.org/10.101 6/j.cjche.2014.06.038

Lin, F., Wang, Z., Zhang, Z., He, Y., Zhu, Y., Shao, J., Yuan, D., Chen, G. and Cen, K. (2020). Flue gas treatment with ozone oxidation: An overview on $\mathrm{NO}_{\mathrm{x}}$, organic pollutants, and mercury. Chem. Eng. J. 382: 123030. https://doi.org/ 10.1016/j.cej.2019.123030

Lin, G., Chen, T. and Tsai, C. (2012). A modified deutschanderson equation for predicting the nanoparticle collection efficiency of electrostatic precipitators. Aerosol Air Qual. Res. 12: 697-706. https://doi.org/10.4209/aaqr. 2012.04.0085
Lu, Q., Yang, Z., Zheng, C., Li, X., Zhao, C., Xu, X., Gao, X., Luo, Z., Ni, M. and Cen, K. (2016). Numerical simulation on the fine particle charging and transport behaviors in a wire-plate electrostatic precipitator. $A d v$. Powder Technol. 27: 1905-1911. https://doi.org/10.1016/ j.apt.2016.06.021

Luo, R., Li, Y., Zheng, C.H., Gao, X. and Fan, J.R. (2015). Numerical simulation of temperature effect on particles behavior via electrostatic precipitators. Appl. Therm. Eng. 88: 127-139. https://doi.org/10.1016/j.applthermaleng.2 014.11 .078

Mizuno, A. (2000). Electrostatic precipitation. IEEE Trans. Dielectr. Electr. Insul. 7: 615-624. https://doi.org/10.110 9/94.879357

Neimarlija, N., Demirdzic, I. and Muzaferija, S. (2011). Numerical method for calculation of two-phase electrohydrodynamic flows in electrostatic precipitators. Numer. Heat Transfer, Part A 59: 321-348. https://doi.org/10.1080/10407782.2011.549080

Ounis, H., Ahmadi, G. and McLaughlin, J.B. (1991). Brownian diffusion of submicrometer particles in the viscous sublayer. J. Colloid Interface Sci. 143: 266-277. https://doi.org/10.1016/0021-9797(91)90458-k

Podlinski, J., Dekowski, J., Mizeraczyk, J., Brocilo, D., Urashima, K. and Chang, J.S. (2006). EHD flow in a wide electrode spacing spike-plate electrostatic precipitator under positive polarity. J. Electrostat. 64: 498-505. https://doi.org/10.1016/j.elstat.2005.10.004

Podlinski, J., Niewulis, A. and Mizeraczyk, J. (2009). Electrohydrodynamic flow and particle collection efficiency of a spike-plate type electrostatic precipitator. $J$. Electrostat. 67: 99-104. https://doi.org/10.1016/j.elstat.2 009.02.009

Schmid, H.J. and Vogel, L. (2003). On the modelling of the particle dynamics in electro-hydrodynamic flow-fields: I. Comparison of Eulerian and Lagrangian modelling approach. Powder Technol. 135-136: 118-135. https://doi.org/10.1016/j.powtec.2003.08.009

Soldati, A. (2000). On the effects of electrohydrodynamic flows and turbulence on aerosol transport and collection in wire-plate electrostatic precipitators. J. Aerosol Sci. 31: 293-305. https://doi.org/10.1016/s0021-8502(99)00055-5

Wang, Y., Gao, W., Zhang, H., Huang, C., Luo, K., Zheng, C. and Gao, X. (2019). Insights into the role of ionic wind in honeycomb electrostatic precipitators. J. Aerosol Sci. 133: 83-95. https://doi.org/10.1016/j.jaerosci.2019.04.011

Yamamoto, T., Abe, T., Mimura, T., Otsuka, N., Ito, Y., Ehara, Y. and Zukeran, A. (2009). Electrohydrodynamically assisted electrostatic precipitator for the collection of lowresistivity dust. IEEE Trans. Dielectr. Electr. Insul. 45: 2178-2184. https://doi.org/10.1109/tia.2009.2031859

Yamamoto, T., Mimura, T., Otsuka, N., Ito, Y., Ehara, Y. and Zukeran, A. (2010). Diesel PM collection for marine and automobile emissions using EHD electrostatic precipitators. IEEE Trans. Dielectr. Electr. Insul. 46: 1606-1612. https://doi.org/10.1109/tia.2010.2049970

Yang, D., Guo, B., Ye, X., Yu, A. and Guo, J. (2019a). Numerical simulation of electrostatic precipitator considering the dust particle space charge. Powder 
Technol. 354: 552-560. https://doi.org/10.1016/j.powtec. 2019.06.013

Yang, Z., Zheng, C., Chang, Q., Wan, Y., Wang, Y., Gao, $X$. and Cen, K. (2017). Fine particle migration and collection in a wet electrostatic precipitator. J. Air Waste Manage. Assoc. 67: 498-506. https://doi.org/10.1080/10 962247.2016.1260074

Yang, Z., Zheng, C., Liu, S., Guo, Y., Liang, C., Zhang, X., Zhang, Y. and Gao, X. (2018a). Insights into the role of particle space charge effects in particle precipitation processes in electrostatic precipitator. Powder Technol. 339: 606-614. https://doi.org/10.1016/j.powtec.2018.08. 056

Yang, Z., Zheng, C., Zhang, X., Chang, Q., Weng, W., Wang, Y. and Gao, X. (2018b). Highly efficient removal of sulfuric acid aerosol by a combined wet electrostatic precipitator. $R S C A d v$. 8: 59-66. https://doi.org/10.1039/ c7ra11520b

Yang, Z., Ji, P., Li, Q., Jiang, Y., Zheng, C., Wang, Y., Gao, X. and Lin, R. (2019b). Comprehensive understanding of
$\mathrm{SO}_{3}$ effects on synergies among air pollution control devices in ultra-low emission power plants burning highsulfur coal. J. Cleaner Prod. 239: 118096. https://doi.org/ 10.1016/j.jclepro.2019.118096

Yang, Z., Zheng, C., Li, Q., Zheng, H., Zhao, H. and Gao, X. (2020). Fast evolution of sulfuric acid aerosol activated by external fields for enhanced emission control. Environ. Sci. Technol. 54: 3022-3031. https://doi.org/10.1021/acs. est.9b06191

Yang, Z., Cai, Y., Li, Q., Li, H., Jiang, Y., Lin, R., Zheng, C., Sun, D. and Gao, X. (2021). Predicting particle collection performance of a wet electrostatic precipitator under varied conditions with artificial neural networks. Powder Technol. 377: 632-639. https://doi.org/10.1016/ j.powtec.2020.09.027

Received for review, July 31, 2020

Revised, October 5, 2020

Accepted, October 12, 2020 